\title{
Spectral features of conjugated bichromophores: porphyrins with curcumin
}

\author{
Aleksandr Starukhin ${ }^{1, *}$, Aleksander Gorski ${ }^{2}$, Tatsiana Pavich $^{1}$, and Valerii Kniukshto ${ }^{1}$ \\ ${ }^{1}$ B.I. Stepanov Institute of Physics, National Academy of Sciences of Belarus, Minsk 220072, Belarus \\ ${ }^{2}$ Institute of Physical Chemistry, Polish Academy of Sciences, Warsaw 01-224, Poland
}

\begin{abstract}
Conjugated bichromophores of porphyrins with natural compound curcumin were synthesized and studied by methods of spectroscopy. The fluorescence spectra of bichromophore consist of four bands covered almost a whole visible spectrum from 450 to $700 \mathrm{~nm}$. A relatively weak interaction between curcumin and metalloporphyrin allows to combine both effects: high generation efficiency of singlet oxygen by metalloporphyrin and an anticancer treatment of curcumin.
\end{abstract}

Design and characterization of novel types of polyheterocyclic compounds have a huge impact on development of methods for the selective transportation of photosensitizers to tumour cells,- a crucial point of photodynamic therapy. Well known, that porphyrins are widely used as effective photosensitizers of singlet oxygen generation in photodynamic processes [1,2]. The idea to design a conjugated bichromophore based on porphyrin macrocycle with a natural compound - curcumin, isolated from the Curcuma longa plant, seems very promising. Nowadays curcumin isomers and derivatives attract a lot of attention of specialists in the field of medicine and biochemistry, primarily as an effective antitumor drugs [3]. Number of publications devoted to the study of the optical and photophysical properties of curcumin [4], as well as its structure modifications have been appeared resently.

In this report we presented of the first results of the synthesis of a structure based on metallocomplexes derivatives of tetraphenylporphyrin linked to curcumin molecule and studying of optical parameters newly-synthetized compounds at ambient and liquid nitrogen temperatures. The structures of Zn-5-(4-aminophenyl) 10,15,20-(triphenyl) porphinato (ZnATPP) and Zn-5-(4-aminophenyl)-1-(4-N-curcumin) 10,15,20-(triphenyl) porphinato (ZnATPP-CURC) are presented on Fig. $1(\mathrm{a}, \mathrm{b})$.

Fluorescence excitation, fluorescence and phosphorescence spectra of ZnATPP and ZnATPP-CURC have been recorded at ambient and liquid nitrogen temperatures. Fluorescence excitation and fluorescence are presented on Fig. 2 (a, b). As evidently from absorption and fluorescence excitation spectra (Fig. 2 a) absorption bands of curcumin and ZnAPTPP are practically coincided in the spectral range of 400-500 $\mathrm{nm}$.

\footnotetext{
* Corresponding author: starukhin@ mail.ru
} 


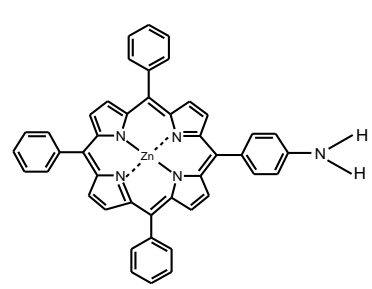

a

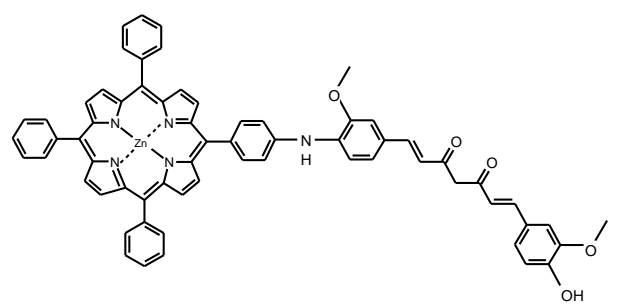

b

Fig. 1. Chemical structures of ZnATPP (a) and ZnATPP-CURC (b) compounds.
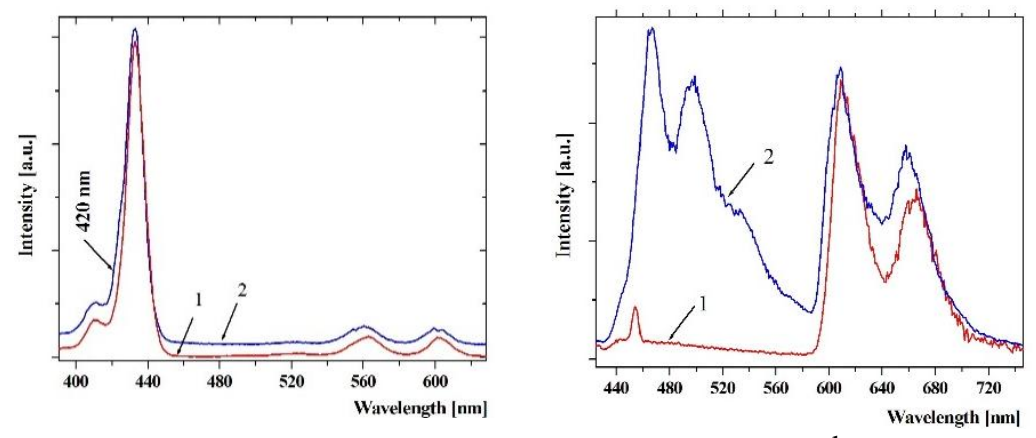

a

b

Fig. 2. Fluorescence excitation (a) and fluorescence (b) spectra of ZnATPP (1) and ZnATPP-CURC (2) in toluene at $77 \mathrm{~K}$.

The absorption bands of ZnATPP-CURC bichromophore exhibits a minor difference compare to ZnATPP in the range of $420 \mathrm{~nm}$. While, the fluorescence spectrum of ZnAPTPP consists from two bands similar to well-known ZnTPP. In contrast, four bands in the spectral range from 450 to $700 \mathrm{~nm}$ (Fig. 2b.2) have been observed in the fluorescence spectrum of ZnAPTPP-CUCR. Two "blue" bands in the spectrum (at 465 and $497 \mathrm{~nm}$ ) refer to the curcumin emission, and two "red" bands at 608 and $659 \mathrm{~nm}$ correspond to the luminescence of ZnAPTPP. Upon formation of bichromophore with curcumin, the bands in the fluorescence spectrum of ZnAPTPP are shifted by more than $2 \mathrm{~nm}$ to the range of short wavelengths. A relatively weak phosphorescence of ZnAPTPP and ZnAPTPP-CUCR were detected with a maximum at about $790 \mathrm{~nm}$.

The complex of ZnAPTPP-CUCR combines a high efficiency of singlet oxygen generation by one part of the complex (ZnAPTPP) with an anticancer effect of curcumin, that can provide to high efficiency of these species as therapeutic agents in the treatment of oncological diseases.

This work was supported by European Union's Horizon 2020 research and innovation program, grant agreement № 645628 and the Belarusian-Polish project № F18PLSHG-003.

\section{References}

1. A. Krasnovsky jr., Biofizika USSR 21, 748 (1976)

2. F. Wilkinson, W. Phillips, J. Phys. Chem. Ref. Data Rev. 22, 113 (1993)

3. V. B. Patel et al., Nutrition and Cancer 62, 958 (2010)

4. K. I. Priyadarsini, J. Photochem. Photobiol. C: Photochem. Rev. 10, 81 (2009) 